\title{
Applications of neutron reflectometry in biology
}

\author{
Yuri Gerelli ${ }^{1, *}$ \\ ${ }^{1}$ Polytechnic University of Marche, Department of Life and Environmental Sciences, Via Brecce \\ Bianche, Ancona, Italy
}

\begin{abstract}
Over the last 10 years, neutron reflectometry (NR) has emerged as a powerful technique for the investigation of biologically relevant thin films. The great advantage of NR with respect to many other surface-sensitive techniques is its sub-nanometer resolution that enables structural characterizations at the molecular level. In the case of bio-relevant samples, NR is non-destructive and can be used to probe thin films at buried interfaces or enclosed in bulky sample environment equipment. Moreover, recent advances in biomolecular deuteration enabled new labeling strategies to highlight certain structural features and to resolve with better accuracy the location of chemically similar molecules within a thin film.

In this chapter I will describe some applications of NR to bio-relevant samples and discuss some of the data analysis approaches available for biological thin films. In particular, examples on the structural characterization of biomembranes, protein films and protein-lipid interactions will be described.
\end{abstract}

\section{Introduction}

The use of neutron reflectometry (NR) in biological sciences has been growing enormously in the last decades. In the field of soft matter and biophysics, the technique was introduced in the '80s for the study of surfactant thin films but NR, as a technique, remained under exploited for biological relevant samples. In the last two decades, use of NR in the filed of biological sciences increased regularly [1]. This was possible mainly because of improvements in instrumentation, sample environment and modeling approaches. These developments allowed investigations of systems of increasing complexity. The fact that NR is a technique applied to interfaces limits the type of samples that can be studied. However, interfaces are ubiquitous in nature and they play important roles in all the living organisms. The main example of a natural interface is represented by the cell membrane, the active barrier separating the interior of cells from the extracellular environment. Because of their nanometric thickness and macroscopic lateral size, cell membranes are a perfect sample for NR experiments. In fact, they represent one of the largest area of application of NR in biology. The first systems investigated were simple solid-supported lipid bilayers (SLBs) composed by a single molecular species and they served as a benchmark to develop sample preparation techniques and analysis methods. Nowadays, SLBs of increased complexity, formed by synthetic or natural molecules, including proteins can be prepared and investigated by NR as it is described in this chapter.

\footnotetext{
*Corresponding author: y.gerelli@univpm.it
} 
Another bio-relevant system to which NR was successfully applied is represented by protein films. Their formation at interfaces is of multiple interest. For example, at the macroscale, engineering of protein coatings is essential to improve the osseointegration of medical implants. At the nanoscale, the formation of protein films around colloidal particles is fundamental in drug-delivery, therapeutic and diagnostic applications. In fact, when nanoparticles are administered into biological fluids they get covered by the so-called protein corona. This protein film represents the interface to the human body and therefore it regulates nanoparticles systemic circulation, biodistribution, and bioavailability.

Finally the combination of proteins and cell membranes represents probably the most investigated class of bio-relevant systems for NR experiments. In fact, interactions of proteins or peptides within and at the surface of cell membranes are fundamental for the understanding of molecular processes involved in diseases, for the development of new drugs and for the design of novel anti-microbial strategies [2-4].

For the study of the above mentioned systems, a great advantage of NR with respect to many other surface-sensitive techniques is a labeling strategy based on the isotopic substitution [5]. This method is referred as contrast variation and in its most common application it exploits the substitution of the naturally occurring hydrogen atoms (protium) by deuterium ones. Lipids [6-8], proteins [9] and many other biomolecules can be either synthesized or naturally produced by microorganisms in partially or fully deuterated forms. The use of hydrogenated and deuterated molecules in the same samples allow to selectively locate individual molecular species and it is therefore particularly suitable for the structural determination of complex biological systems.

Within the framework of biological application of NR, I will briefly describe the main sample preparation approaches currently available and the analysis methods that can be exploited for the analysis of extremely flat and homogenous or rough and diffuse biological thin films. In the second part of the chapter I will give a series of examples to highlight what type of systems can be studied and what information can be obtained.

\section{Sample preparation approaches}

As described by Cousin and Fadda in the chapter "An introduction to neutron reflectometry", NR requires the presence of an interface onto which samples, in the form of thin films, can be deposited or adsorbed. Typical interfaces for biological samples are the solid-liquid, liquid-air and solid-air. At these interfaces samples can be deposited by several techniques such as adsorption from a liquid phase, drop-casting and spin-coating, Langmuir-Blodgett and Langmuir-Schaefer deposition methods $[10,11]$.

Adsorption from a liquid subphase is a well established method to create self-assembled films at an interface. Among several examples it might be worth mentioning the case of vesicles fusion, a technique used to obtain a single lipid bilayer at a solid-liquid interface [12] or the adsorption from bulk in which a molecular film is created by the natural tendency of the molecules of interest in partitioning between the aqueous phase and the interface $[13,14]$. In both cases the bulk liquid phase is enriched with the molecules of interest and, as a result of a kinetic process, these molecules can self assemble into an interfacial thin film.

Spin-coating is another well established technique to deposit in a controllable way molecules at a solid-air interface. A wanted amount of a solution containing the molecules of interest can be added drop-wise onto a solid substrate which rotates at a controlled rate. While the solvent is either ejected as an effect of the rotation or evaporates, the molecules it contains can self-assemble or organise at the interface. This approach is typically used to create multilayered structures $[15,16]$ that can be then either studied at the solid-air interface or exposed to an aqueous phase in solid-liquid geometry. 
Langmuir-Blodgett and Langmuir Schaefer are two deposition techniques in which monolayers prepared at the air-water interface (typically using an equipment called Langmuir trough) can be transferred onto solid substrates. These techniques are widely used to prepare single solid supported bilayers for which is crucial to control the composition of the individual monolayers, as in the case of asymmetric systems [17, 18]. In addition, these two techniques are essential to prepare lipid and surfactant mono- or multi-layer systems as well as double or multi-bilayers systems with an accurate control of the number of bilayers and of their composition $[19,20]$.

\section{Modeling method for biological problems}

One of the main bottleneck limiting the use of NR in biological sciences is the necessity of adapting or developing new modeling approaches that could be used to describe the structure of samples of increasing complexity. In the following I will review a series of modeling approaches that can be used for the analysis of biological systems at interfaces. All of them are based on the computation of a theoretical scattering length density (SLD) profile describing the distribution of molecular groups or atomic species along the vertical direction with respect to the interface. The distance from this interface is typically indicated as $z$ and expressed in Ångströms or nanometers. In general, from an SLD profile it is possible to compute the corresponding theoretical reflectivity curve, $R(Q)$, to be refined against the experimental data. This leads, in turns, to the refinement of the SLD profile that can be therefore interpreted to obtain a correct description of the structure of the sample. In fact, since the SLD is determined by the number density of atomic nuclei (see chapter "An introduction to neutron reflectometry" for the definition of SLD), these profiles are directly linked to the distributions of the components in the sample and therefore describe the vertical structure of interfacial thin films. By applying the contrast variation method, the scattering signal of one or more components can be altered resulting in altered SLD profiles. In this way, the location of selected components within the sample can be highlighted.

In the following I will briefly describe the approaches that are commonly used for the modeling of NR data of bio-relevant systems.

\subsection{Slab models}

The slab model is the oldest model developed for the analysis of NR data which can be explicitly used in the calculation of reflectivity from stratified interfaces [21, 22]. In this model a thin film is described as discrete series of layers. In this context, a layer is defined as a portion of the sample characterized by a SLD value that differs from those of the neighbouring regions along the $z$ direction. An interface is therefore the line in space where a change in SLD takes place. Since reflectometry probes only the vertical direction with respect to an interface, SLD changes must occur along the $z$ sample axis while lateral homogeneity is preserved. This implies that even if the sample is physically not stratified (for example it is composed by a single molecular layer) it might be modeled as a series of slabs if the $\operatorname{SLD}(z)$ changes within the sample. This is, for example and among many others, the case of a surfactant layer at the air-water interface [23] or of a supported lipid bilayer (see Figure 1). In general, each slab is characterized by three structural parameters, namely thickness, SLD and interfacial roughness. In some cases, especially upon the use of the contrast variation technique [5], the total SLD of a given layer can be redefined as a function of the SLD of the molecules composing the layer and of the volume fraction of solvents present in the same region. This redefinition is commonly used in the case of thin films in which hydration plays 


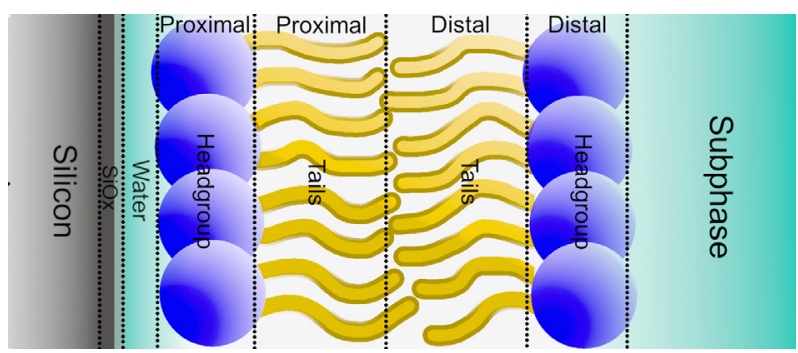

Figure 1. Schematic representation of the slab model used to describe a SLB on the top of a silicon substrate. Reprinted with permission from Ref. [24].

an important role [25] and for mixed systems [8]. In general, the total SLD $\rho$ of a slab composed by $n$ different chemical groups or materials can be expressed as

$$
\rho=\sum_{i=1}^{n} \Phi_{i} \times \rho_{i}
$$

where $\Phi_{i}\left(\sum_{i=1}^{n} \Phi_{i} \equiv 1\right)$ is the volume fraction of the i-th component and $\rho_{i}$ is its SLD. Equation 1 is particularly useful in case of hydrated samples where one of the components represents hydration water.

The slab model is rather general and can be applied to a large variety of systems. It is, in fact, implemented in the most common software applications devoted to the analysis of NR data originated from soft or biological samples [25-29]. However, the slab model might fail if the interfacial roughness between subsequent slabs is too large (approximately larger than the slab thickness) or if the sample is laterally and vertically inhomogeneous. In these cases, alternative approaches should be used and some of them are described in the following sections.

\subsection{Functional component groups}

As reported in the previous section, the main limitation of the slab model is the description of (vertically, along z) diffuse layers. This can be the case, among several others, of polymer brushes deposited onto surfaces [30-32] or of inhomogeneous systems [33], for which a description through a limited number of well defined layers might fail. As reported in the cited literature, these systems can be modeled by a combination of slabs, for the homogeneous regions (if any) of the sample, and by functional distributions, describing volume fraction profiles, for the inhomogeneous ones. In many cases, the shape of the functional profiles to be used can be retrieved from theoretical predictions while their parameters can be varied during the data analysis. For example, a lipopolysaccharide (LPS) monolayer deposited at the air water interface can be modeled as described in Figure 2, where the polysaccharide chains are described by a stretched exponential function [33] while the hydrocarbon tails and the oligosaccharide core are described sufficiently well by two slabs. The same approach was used for a large variety of systems [30-32] in which at least one component can be described as a polymer brush. Volume fraction profiles can be converted into SLD profiles simply by weighting them according to the nominal SLD values associated to the desired material or chemical species. Similarly, from a given volume fraction profile several SLD profiles can be obtained to simulate the effect of contrast variation i.e. the change of a given SLD value 

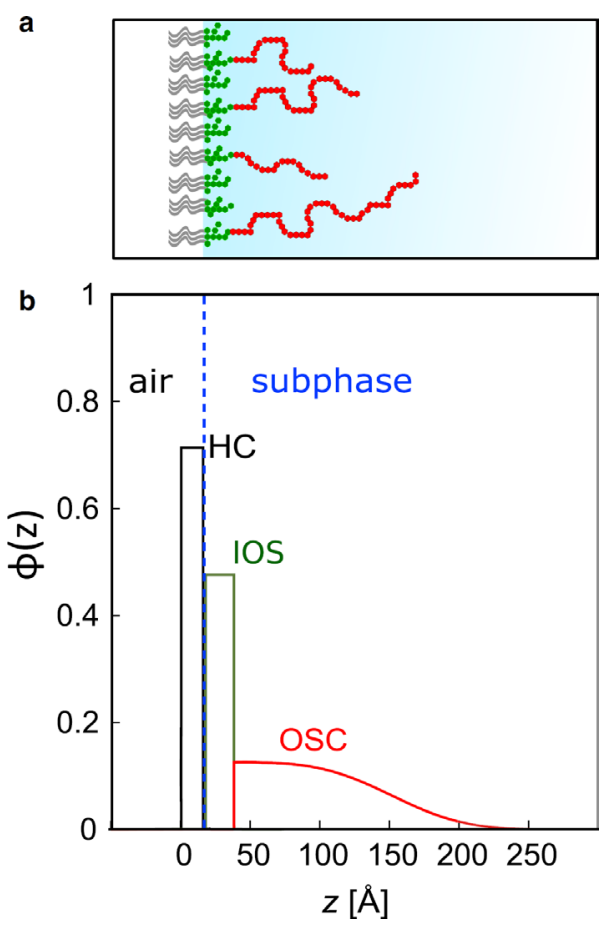

Figure 2. (a) Schematic representation of a lipopolysaccharide monolayer at the air-water interface. (b) Theoretical model used to describe the volume fraction distributions of the LPS groups at the interface. The components are hydrocarbon chains (HCs), inner oligosaccharides (IOSs) and O-side chains (OSCs). Reprinted from Biophysical Journal, 116, S. Micciulla, Y. Gerelli and E. Schneck, Structure and Conformation of Wild-Type Bacterial Lipopolysaccharide Layers at Air-Water Interfaces, 12591269, Copyright (2019), with permission from Elsevier.

taking place because of isotopic substitution. Once the SLD profiles are generated, the optimization procedure of the structural parameters is exactly the same as the one described for the slab model. In fact, to calculate the corresponding reflectivity curve the functional SLD profile is sliced in finite-size slabs (typically small enough to reproduce accurately the original curve) and used within the standard Parratt's or Abelès' formalisms.

A similar approach was also developed for the analysis of SLBs, not because of the failure of the slab model but because of the necessity to compare models obtained from NR measurements on flat membranes to those obtained from vesicle samples by small angle X-ray and neutron scattering data [34-36]. In this approach, a lipid molecule is modeled as the sum of the probability distributions of its component groups calculated along the $z$ direction (parallel to the lipid acyl chains). The components could be (for example): choline methyl groups, phosphate $+\mathrm{CH}_{2} \mathrm{CH}_{2} \mathrm{~N}$, carbonyl-glycerol $(\mathrm{GC})$, hydrocarbon methylene groups $\left(\mathrm{CH}_{2}\right)$ and methyl groups of hydrocarbon chains $\left(\mathrm{CH}_{3}\right)$. Such a model for a SLB at a solid-liquid interface is given in Figure 3. In this case, all the groups are represented by functions whose parameters can be adjusted, within the appropriate constraints, and refined during data analysis. The only component not described by an analytical function is the probability distribution of water, that is calculated as the complement to unity of all the other probabilities. This allows the model to reproduce not only perfect SLBs but also systems with a non uniform and partial coverage. The SLD profile is obtained by the sum of all the distribution functions 


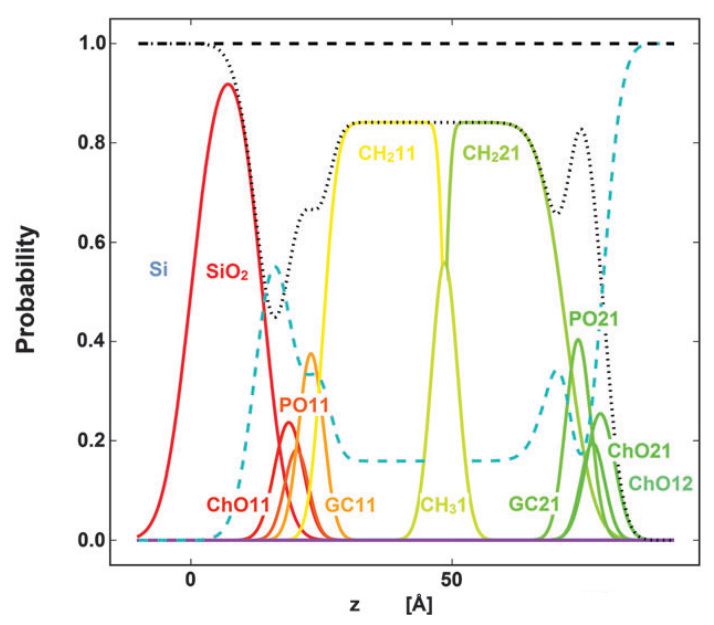

Figure 3. Scheme of component group probability distributions within the SLD profile model of a supported lipid bilayer. The components are: bulk silicon $-\mathrm{Si}$, a silicon dioxide layer $-\mathrm{SiO}_{2}$, hydrocarbon chain region $\mathrm{CH}_{2}$, water $-\mathrm{H}_{2} \mathrm{O}$ choline $-\mathrm{ChO}$, phosphate $-\mathrm{PO}$, carbonylglycerol - $\mathrm{GC}$ and methyl groups - $\mathrm{CH} 3$ ). $\mathrm{XY}$ in the group names label are references for the leaflet in which the groups are. Reproduced from Ref. [36] with permission from the Royal Society of Chemistry.

weighted by the nominal SLD values associated to a given molecular group. This profile can be sliced into finite-size slabs and reflectivity calculated as described in section 3.1. Examples of applications of these approaches to biological systems are given in Section 4.2.2.

\subsection{Simulation-based methods}

In soft matter, biophysics and biology, the use of simulations for the analysis of large scale structures measured by means of neutron scattering experiments is well established for most of the scattering techniques available [37-40]. In the context of neutron reflectometry applied in biological sciences only in the last two decades, the use of MD simulations to support the interpretation of experimental data gained a reasonable traction. As already mentioned in the previous sections, the result of an analysis of NR data consists in scattering length density or in volume fractions profiles. Similar profiles can be obtained from the output of computer simulations and therefore a comparison between experimental and computational results is rather straightforward. The use of computer simulations to complement or cross-validate the analysis of experimental NR data has been reported for several systems, including proteins at interfaces [41], membranes and peptide-membrane interactions [28, 42, 43].

More recently, different groups have started to use MD simulations not only as a complementing information but also as a tool for the analysis of NR data. This was possible because of the improvements made in the quality of parameters sets in MD simulations. An example of the importance of the force-field for a correct reproducibility of experimental data has been given in the work by Koutsioubas on the phase transition of a solid-supported lipid bilayer [44]. One of the first remarkable examples of combination of traditional modeling approaches and numerical simulation was described by Heinrich and Lösche [45]. In their work, they demonstrated that it is possible to determine the out-of-plane localization of transmembrane proteins in lipid membranes with sub-nanometer precision by using all-atoms simulations to interpret NR data. 
In a more recent work, McCluskey et al. [46] investigated whether the use of atomistic simulations are necessary to reproduce accurately experimental reflectometry data or whether coarse-grained representations are sufficient. By the comparison between the results obtained using different MD potential models and those originated from a traditional slab description of a monolayer sample, the authors concluded that discrepancy between simulations and experimental data are likely to be observed if the coarse-grained representation is too simplified. As the resolution of the potential model increased, the agreement with experimental data improved. However, any coarse-grained representation could describe with the same goodness as a traditional slab model, the reflectivity curves.

Concerning bio-systems, Darré et al. made a direct comparison not only between SLD profiles calculated from simulations and experiments but also between reflectivity curves and volume fraction profiles [47] by using the NeutronRefTools plugin available in the VMD molecular visualization and analysis software [48]. Information from MD simulations were extracted from both coarse-grained and all-atoms molecular dynamics trajectories and reflectivity and SLD profiles were extracted by slicing the MD trajectory into a discrete number of slabs as shown in Figure 4.

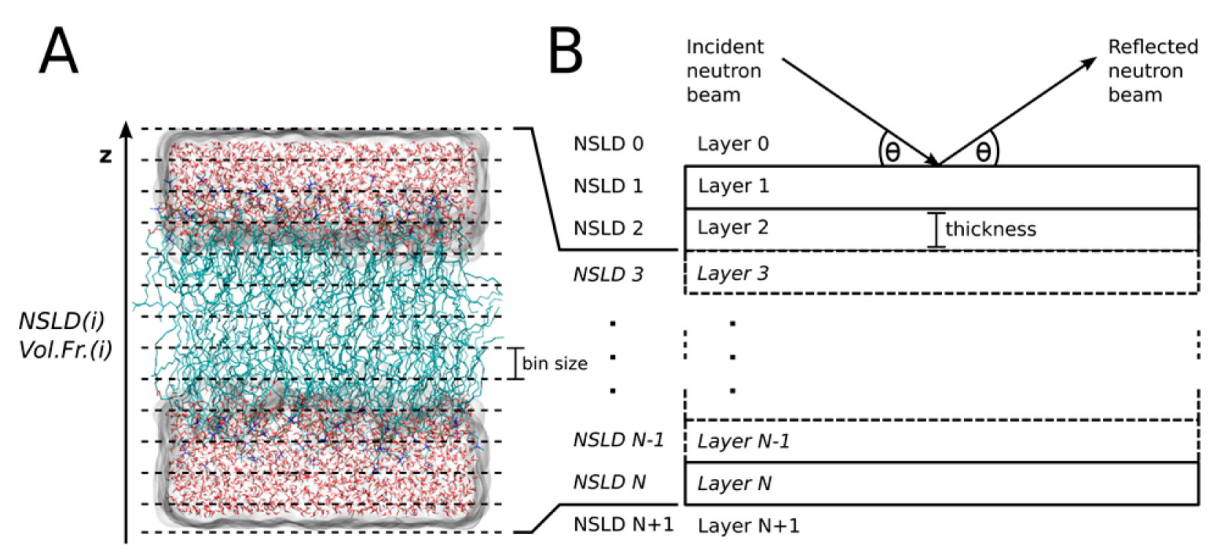

Figure 4. Definition of a slab model for the calculation of reflectivity and SLD profiles from MD trajectories. Reprinted with permission from Ref. [47]. Copyright (2015) American Chemical Society.

\section{Examples of applications}

This section contains some examples selected from articles already published in literature highlighting the possibilities offered by NR in the field of biological sciences. These examples were chosen to compare the approaches available for the analysis of bio-systems, the variety of samples that can be investigated and the different information that can be obtained by the analysis of NR data. In any case, this represents a very restricted subset of applications of NR to biological systems and most of them were selected for the quality of the work presented and for their pedagogical aspects.

\subsection{Biological membranes}

Bio-membranes are the molecular assemblies at the basis of life. They are chemically diverse and formidably complex [49-51]. Cell membranes are not only passive barriers that separate 
the interior of cells from the outside environment protecting the integrity of the cell. They are indeed active barriers able to perform different and fundamental processes such as control of selective exchange of substances between inner and outer cellular environments, regulate cell growth and recognize external stimuli [52]. Because of their nanometric thickness, they are perfect systems to be studied by means of neutron reflectometry [53]. Their study is fundamental for several aspects; they can be used to improve our knowledge on diseases involving cell membranes alterations, to develop new drugs and to understand how vital processes are regulated.

"Simple" lipid bilayers composed by a limited number of synthetic phospholipid species are systems investigated routinely and their structural characterization can be obtained by standard NR measurements. However, these simple systems remain of interest for the investigation of collective fundamental physical properties as fluctuations [54, 55], phase transitions $[24,44]$ or structural dynamics $[17,56]$.

In the last ten years, greater interest has been growing towards the investigation of more complex membranes composed by several constituents $[57,58]$ or obtained from natural systems [59].

Of particular interest is the case of reconstituted Gram-negative bacterial membranes. Gramnegative bacteria are of biomedical and technological interest because of their increasing antibiotic resistance. In Gram-negative bacteria, the outer membrane (OM) acts as a barrier preventing antibiotics to enter in the bacteria and therefore hinders their activity. For this reason, a structural investigation of the $\mathrm{OM}$ is of particular interest either in presence or absence of antimicrobial molecules. Considering applications of NR to these systems, Clifton et al. [60] fabricated an accurate in vitro model of the E. coli envelope. In particular, they

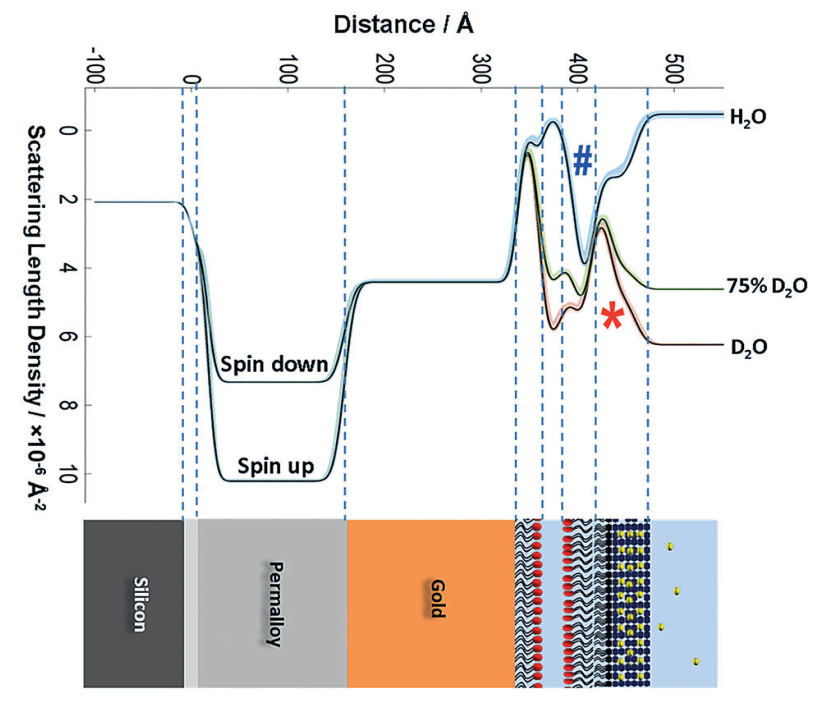

Figure 5. SLD profiles (A) of the OM model in different contrasts together with a schematic representation (B) of the OM model. The permalloy layer was necessary for the use of polarised neutrons. The strong peaks of the deuterated DPPC tails versus $\mathrm{H}_{2} \mathrm{O}(\#)$ and the non-deuterated LPS tails versus $\mathrm{D}_{2} \mathrm{O}$ $(*)$, which confirm asymmetry, are evident. Reprinted with permission from Ref. [60].

produced a model system, with a very complex architecture, closely recreating the natural behavior of the E. coli OM. The main feature of an OM resides in its asymmetric structure, with lipopolysaccharide (LPS) located in the outer leaflet of the membrane (the one facing the 
extracellular environment) and phospholipids facing the interior of the cell. This profound asymmetry creates a uniquely impermeable layer that makes OM resistant against the uptake of molecules such as antibiotics [61]. Samples were prepared by Langmuir-Blodgett and Langmuir-Schaefer deposition techniques in order to have a full control on the composition of the inner and outer leaflets of the membrane. The authors deposited the asymmetric OM onto a metallic substrate fractionalised with a self-assembled phosphatidylcholine monolayer. In this way, the OM floats on the top of a water cushion and is decoupled from the solid substrate. To enhance the presence of an asymmetric structure, deuterated phospholipids were used as components on the inner OM leaflet. The authors used neutron reflectometry to reveal, with sub-nanometer resolution, the asymmetric structure of the model OM. The asymmetric structure of the OM mimicking system is shown in Figure 5 in terms of its SLD profiles determined form the analysis of NR data. As reported by the authors, the analysis of such a complex system is however easier than the creation of an accurate in vitro systems, which is technically challenging. The structure of the LPS leaflet compared well with the one determined for LPS monolayers at the air-water interface [62]. The structure of the bilayer resulted also compatible with a model generated from atomistic molecular dynamics simulations and reported in [60]. The stability of the model OM was tested under different solution conditions, including presence and absence of divalent cations, EDTA (a chelating agent) and antimicrobial peptides. It is known that in vivo, EDTA removes divalent cation and promotes a LPS depletion form the OM, leading to the appearance of phospholipids in the outer leaflet [63]. In their in vitro model, Clifton et al. observed that bilayer asymmetry was reduced by approximately $20 \%$ upon the sequestration of $\mathrm{Ca}^{2+}$ by EDTA, confirming thus the possibility to replicate the in vivo OM behaviour.

The authors monitored also the effect of antimicrobial proteins, such as lactoferrin, produced by the human immune system. Lactoferrin is a cationic protein and it is supposed to act upon the OM through electrostatic interactions, disrupting divalent-cation bridges between LPS molecules, causing LPS release from the membrane. Since the SLD of lactoferrin differs for that of LPS and phospholipids, it is possible to use NR to localise proteins within the OM structure (see Figure 6). The authors could observed that because of the interaction with

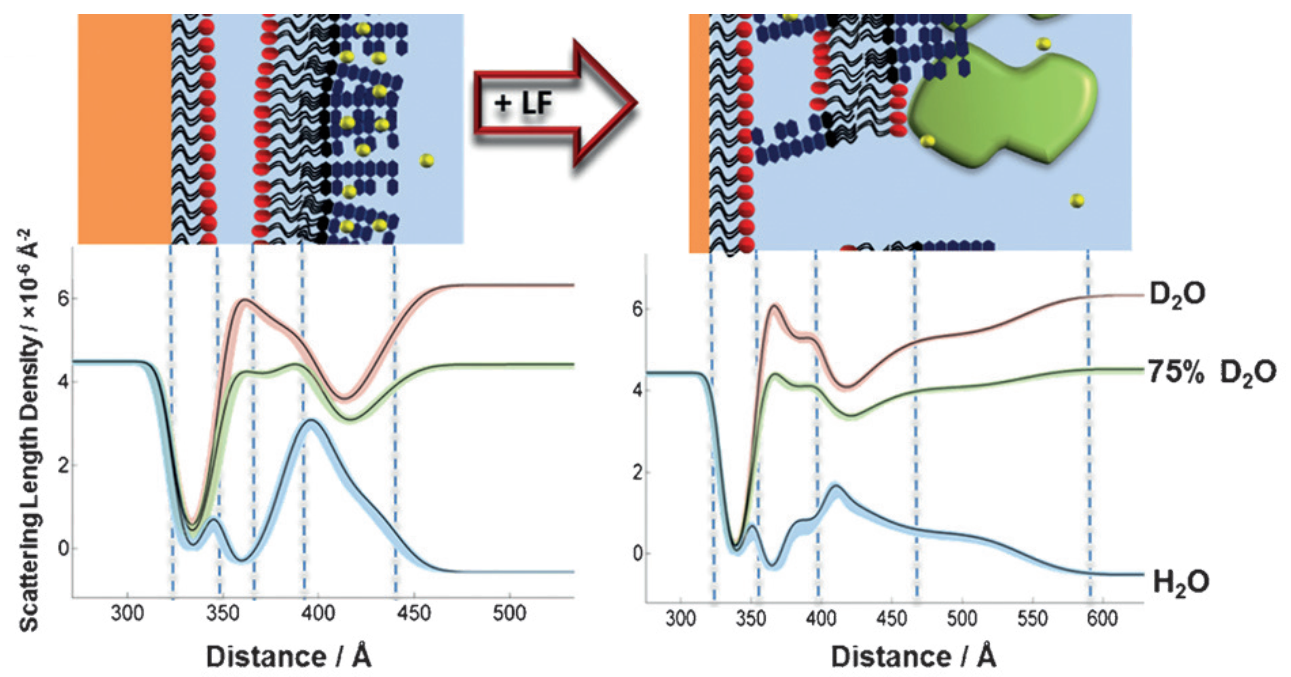

Figure 6. SLD profiles and schematic representation of the OM model before and after addition of lactoferrin. Reprinted with permission from Ref. [60]. 
lactoferrin, the OM coverage and asymmetry were noticeably reduced. At the same time, the thickness of the LPS core region increased by $90 \AA$ suggesting that proteins were bound to the bilayer with their major axis parallel to the membrane normal. All together, the authors could provide the first structural picture of lactoferrin disrupting the OM.

\subsection{Biomolecules at interfaces}

Protein adsorption at interfaces represents a very active research area for which neutron reflectometry plays an important role. In fact, while other experimental techniques as QCM-D (Quartz Crystal Microbalance with Dissipation monitoring), ellipsometry, film balance, AFM (Atomic Force Microscopy) and SPR (Surface Plasmon Resonance) can be used to determine the amount of absorbed material and the kinetics of absorption, NR can provide a characterization of the organisation of protein molecules at interfaces. Among many cases, the use of NR is particularly advantageous for the study of i) decorated interfaces promoting protein adsorption at different distances from the interface (for example in presence of polymer brushes) and of ii) adsorption leading to the formation of multilayers.

\subsubsection{Proteins at a solid-liquid interface}

In the framework of the investigation of protein stability in chromatography processes, Bracewell et al. [64] used NR at a solid-liquid interface to study the adsorption of immunoglobulin $\mathrm{G}(\mathrm{IgG})$ at various solid-liquid interfaces. We report here the case of adsorption on a pristine silicon-water interface. It is worth recalling that silicon substrates used in NR are typically characterized by the presence of a naturally-growing silica layer on their surface which can be rendered hydrophilic by cleaning or etching processes. The interface of interest is therefore silica and not that of a pure silicon crystals. The surface of the solid substrate was put in contact with an aqueous buffer in which IgG was injected. Measurements were performed using both $\mathrm{D}_{2} \mathrm{O}$ and $\mathrm{H}_{2} \mathrm{O}$-based buffers in order to apply the contrast variation method [5]. Data were analysed according to a slab model introduced briefly in Section 3.1. The resulting SLD profiles, together with the relative reflectivity curves are displayed in Figure 7 for the pristine silicon substrate, adsorbed $\mathrm{IgG}$ and bound $\mathrm{IgG}$ remaining at the interface after a rinsing step. The SLD profiles were interpreted in terms of the amount (volume fraction) of protein present in each of them following the relation

$$
\rho_{j}=\Phi_{w, j} \rho_{w}+\Phi_{I g G, j} \rho_{I g G}
$$

where $\rho_{j}$ is the total SLD of the $\mathrm{j}$-th layer, $\rho_{w}$ and $\rho_{I g G}$ the SLD of buffer and of IgG. The volume fractions of buffer and IgG in the j-th layer are $\Phi_{w, j}$ and $\Phi_{I g G, j}$ respectively $\left(\Phi_{w, j}+\right.$ $\Phi_{I g G, j} \equiv 1$ by definition). Equation 2 is simply the two-components version of Equation 1 . By evaluating $\Phi_{I g G}$ in each slab and comparing the slabs thickness to the structure of IgG in solution, the authors could conclude that the layer closest to the silica surface represented a population of IgG molecules adsorbed in a "flat on" orientation while the outer layers were ascribed to a sparse self-associating IgG layer in a flat to side-on orientation. The effect of the rinsing step, that caused the removal non-bound $\mathrm{IgG}$ molecules, was also visible from the changes in the SLD profiles for large $z$ values.

\subsubsection{Antibodies on PEG-brushes}

Resistance to protein adsorption onto surfaces is of large interest for biotechnological and pharmaceutical applications. It is known that polyethylene glycol (PEG) chains can resist 


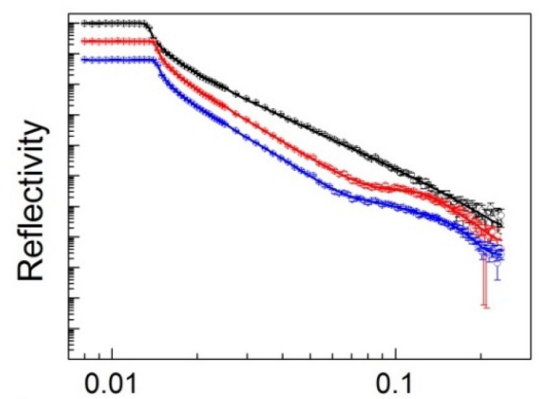

A

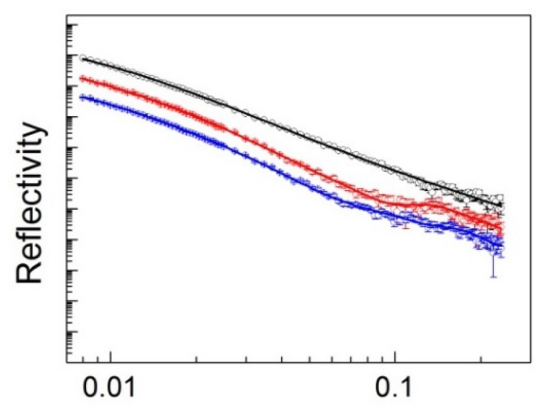

B

$Q\left(\AA^{-1}\right)$

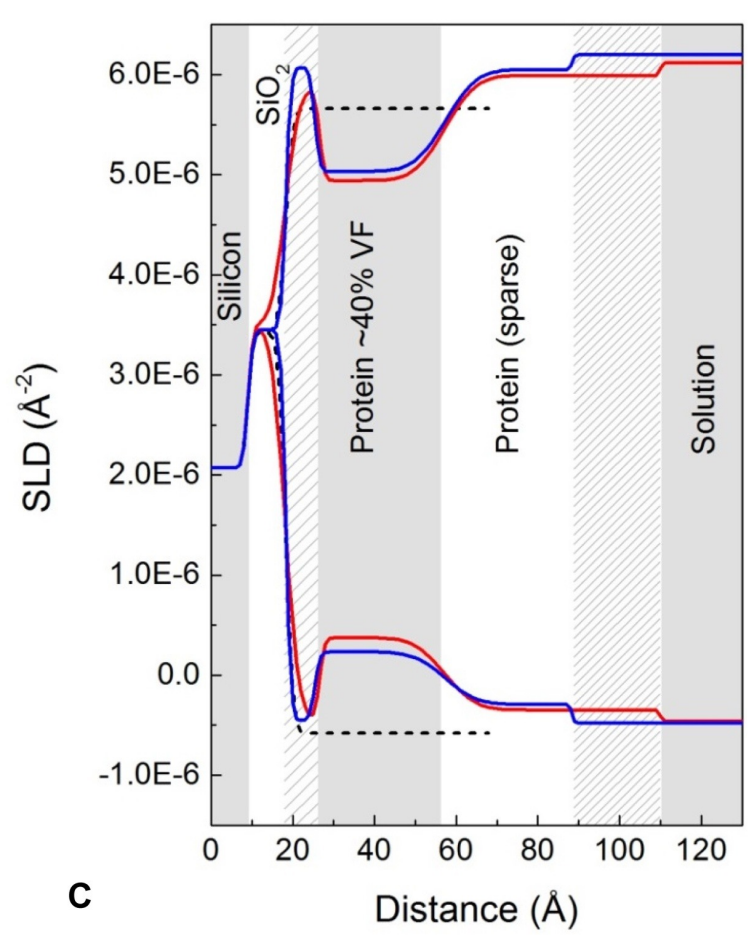

Figure 7. A-B) Reflectivity curves for the pristine silica (black) and for the sequential IgG adsorption (red) and rinse (blue) steps for $\mathrm{D}_{2} \mathrm{O}(\mathrm{A})$ and $\mathrm{H}_{2} \mathrm{O}$ (B) based buffers. Curves are offset to improve their visibility. C) Scattering length density profiles as a function of the distance $z$ from the substrate surface obtained from the $R(Q)$ curves shown in panels A and B. Reprinted from Ref. [64] under the Creative Commons Attribution License (CC BY).

protein adsorption $[65,66]$. In order to understand the molecular mechanisms at the basis of successes and failures of PEG-based surface coatings, Schneck et al. [30] used NR to evidence ternary adsorption of antibodies (in particular of anti-PEG Immunoglobulin G) on methoxy terminated PEG brushes. For this purpose, the surface of a silicon crystal was modified by the deposition of an octadecyltrichlorosilane (OTS) layer onto which lipid monolayers anchoring the PEG brushes were deposited by the Langmuir-Schaefer technique. The surface was exposed to a water-based buffer in which IgG antibodies (Ab) were dispersed. NR measurements were performed after incubation and rinsing steps and collected in different H/D contrasts. NR data were then analysed using a component groups approach similar to the one described in Section 3.2. Figure 8 shows the resulting volume fraction distributions together with a pictorial sketch describing the structure of the investigated system. NR experiments were performed for different brushes grafting density and the authors could evidence a clear increase of the adsorbed amount of $\mathrm{Ab}$ as the grafting density increased. From the analysis of the volume fraction profiles, the authors could determine the type of Ab adsorption, concluding that the latter took place within the PEG brush because of an attractive interaction between Abs and PEG (ternary adsorption). Moreover, a more detailed interpretation of the volume fraction profiles indicated that the antibodies adsorbed in an inverted "Y" configuration, interacting mainly with the terminal part of the PEG chains. 

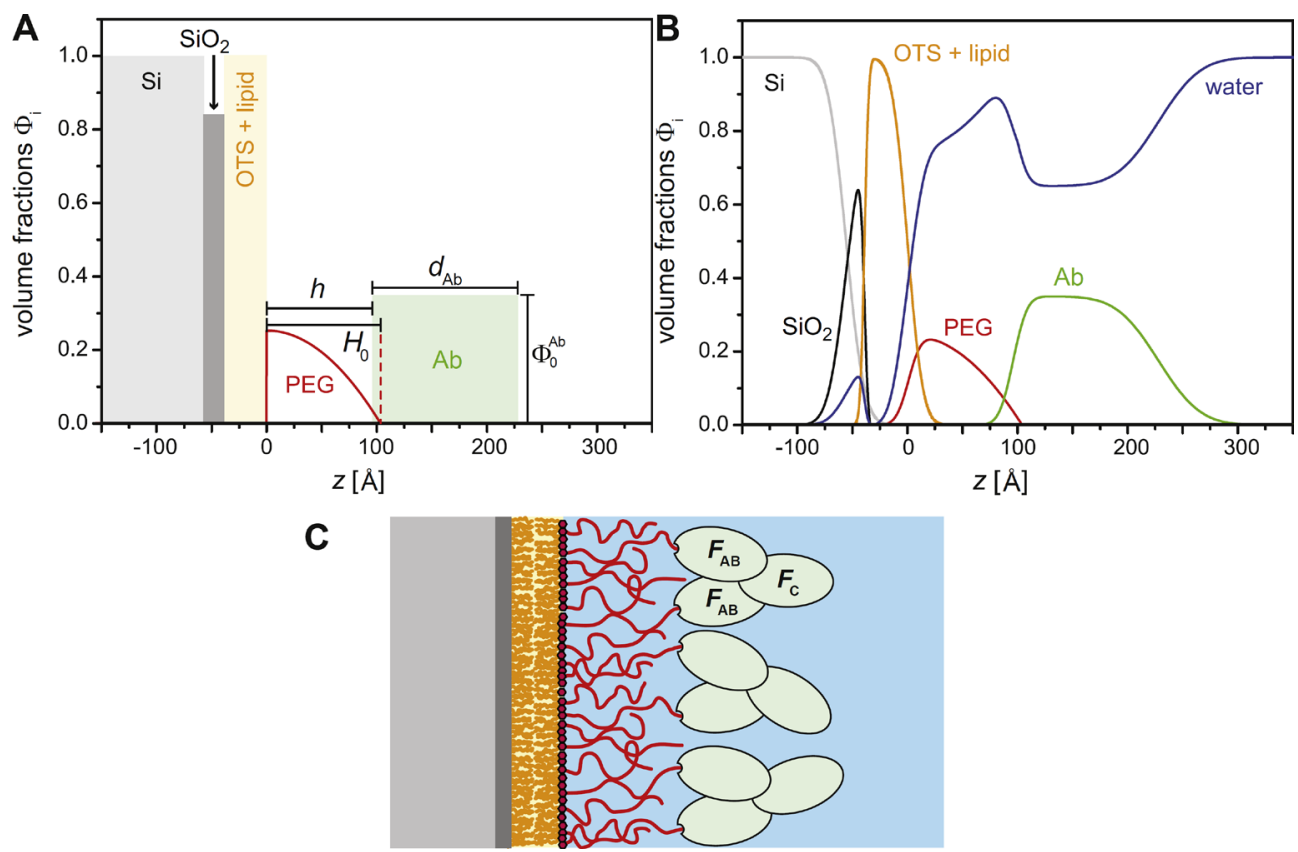

Figure 8. A) Idealised volume fraction model before optimisation, B) optimised volume fraction distribution obtained from the analysis of NR data and C) pictorial representation of the equilibrated structure showing Ab binding. Reproduced from Ref. [30] with permission from Elsevier.

\subsection{Interactions with membranes}

Interactions of small natural and synthetic molecules with lipids permeate our daily life. At the level of the cell membrane, these interactions are essential for the proper functioning of living organisms and play important roles in diseases, in their diagnosis and in therapeutic processes. They are also or primary interest in food sciences, being lipid molecules among the most common components of food. Lipid monolayers can be found as stabilisers at many interfaces and in emulsions and therefore they have a major role in food preservation, food digestion and fat uptake. Among all these several possible examples, this section will be focused on two important studies reporting on the cytotoxicity of amyloid polypeptide and on the antimicrobial action of a well known drug, Amphotericin B.

\subsubsection{The case of an amyloid polypeptide}

As described in Sections 4.1 and 4.2, lipids and proteins films represent typical samples investigated by NR. Increasing attention has been devoted, in the last 10 years, to the study of the interactions between these two biomolecules. In fact, protein-membrane interactions are of general great interest; they are at the basis of many diseases (including some of high societal impact as Alzheimer's and Parkinson's) and, at the same time, they can be exploited for developing novel antimicrobial and therapeutic strategies. Peptide-membrane interaction mechanisms have been the subject of numerous studies and debates in the past decades and are still a puzzle. Neutrons scattering techniques, and in particular neutron reflectometry, have been used in all these contexts. Among several possible examples, the case of amyloid peptides involved in neurodegenerative and systemic diseases [67] was chosen. Martel et al. 
[68] used NR, together with other experimental techniques, to investigate the amyloidogenicity and cytotoxic mechanisms of an amyloid polypeptide (IAPP) involved in type 2 Diabetes Mellitus. In order to identify which peptide fragments (genes) are promoting interactions with membranes (and therefore involved in cytotoxic effects related to the type 2 diabetes Mellitus), structural changes induced on model-membranes by the human peptide were compared to those induced by rat and mutagen peptides. This comparison was performed since rats are not affected by this disease even if they host an amyloid polypeptide characterized by a genetic sequence very similar to that of the human one. By performing NR experiments, the author could quantify and localise the structural changes induced in a solid supported bilayer (SLB) as reported in Figure 9. The SLD profiles shown in Figure 9 refer to the pep-
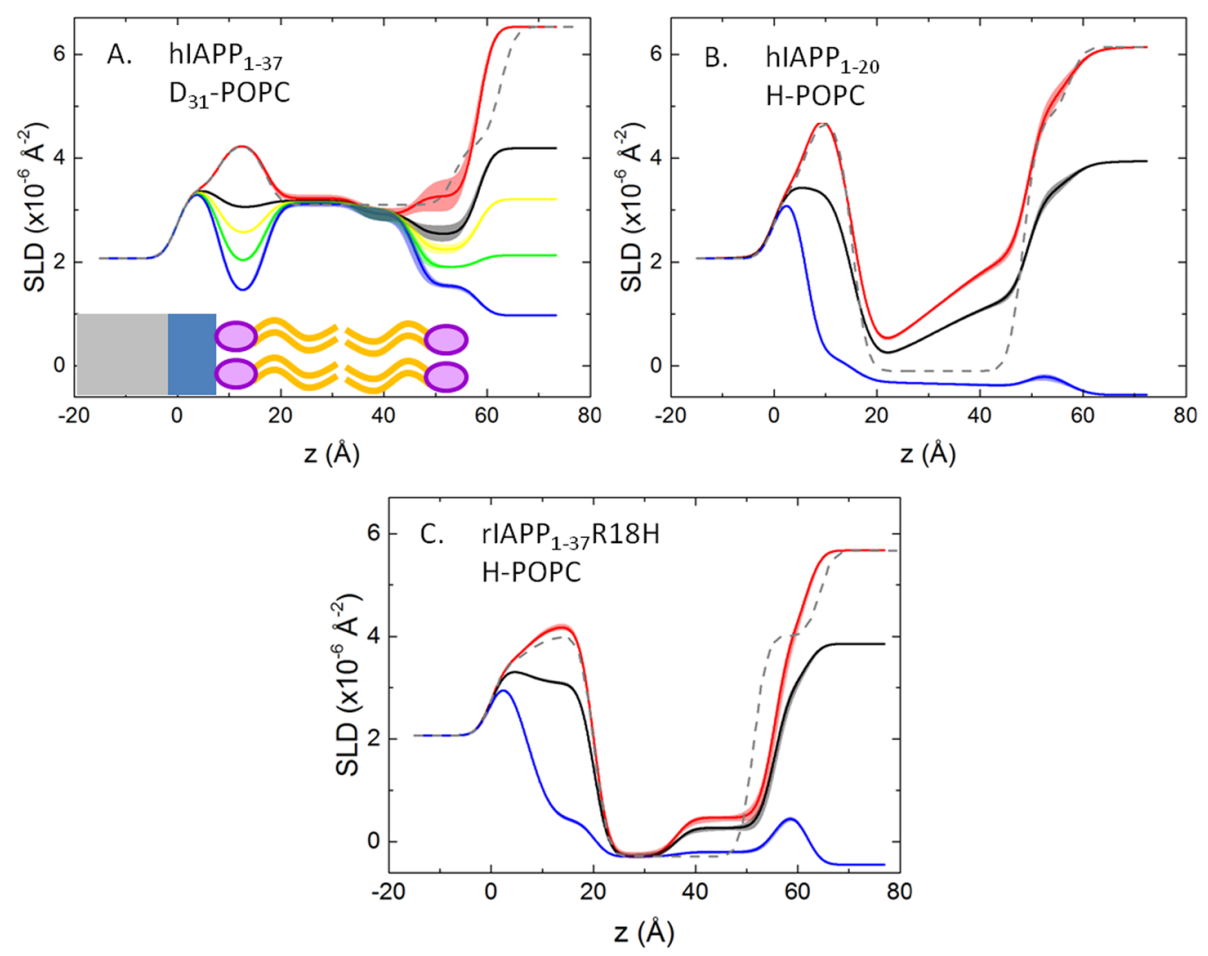

Figure 9. SLD profiles obtained from the analysis of NR curves recorded for SLBs (A $d_{31}$ POPC, B-C POPC) in presence of different peptides and at different contrasts: $0 \% \mathrm{D}_{2} \mathrm{O}$ in blue, $38 \% \mathrm{D}_{2} \mathrm{O}$ in green, $52 \% \mathrm{D}_{2} \mathrm{O}$ in yellow, $66 \% \mathrm{D}_{2} \mathrm{O}$ in black, $100 \% \mathrm{D}_{2} \mathrm{O}$ in red. The SLD curves of pristine SLBs in $100 \%$ $\mathrm{D}_{2} \mathrm{O}$ buffer are also shown as dotted lines. Reprinted with permission from Ref. [68]. Copyright (2016) American Chemical Society.

tides that were found to induce alterations in the bilayer structure. They indicated that the full length human IAPP (hIAPP $1-37$ in panel A in Figure 9) interacted only with the outer leaflet (see Figure 1) of the SLB. The change in the profiles was interpreted as a removal of phospholipid molecules. A similar, but larger, effect was observed for the mutagen rat peptide rIAPP $_{1-37} \mathrm{R} 18 \mathrm{H}$ (panel $\mathrm{C}$ in Figure 9), in which the residue 18 of the original rat peptide was exchanged for the human one. This simple modification induced an important peptidemembrane interaction, otherwise absent for the full length rat peptide [68]. The largest effect, indicating the largest cytotoxicity, was observed for the fragment corresponding to the first 20 residues of the human IAPP ( hIAPP $_{1-20}$ in panel B in Figure 9). In this case the membrane 
was partially destroyed as indicated by the large split, in the z-range corresponding to the hydrocarbon region, between the SLD profiles measured in different contrast conditions for the same sample. Such a split indicated water permeation of the SLB and it was related to the formation of large, macroscopic pores. By comparing these results with those obtained from other experimental techniques and simulations, the authors described a new scenario in which the peptide toxicity is not caused by the formation of amyloid fibers (on the contrary to other famous disease as Alzheimer's) but by the effect of a membrane-disturbing peptide oligomer, that lead to the formation of pores in the membrane. For this class of peptides, already existing hypotheses on the link between the formation of large amyloid aggregates and self-protection mechanisms inhibiting peptide toxicity were confirmed. Indeed, the toxic portions of the peptide were isolated within the aggregates and their interaction with cell membranes was, in this way, avoided.

\subsection{The case of Amphotericin B}

Amphotericin B (AmB) is a highly toxic antifungal drug used to treat systemic fungal infections. It is now widely accepted that $\mathrm{AmB}$ action is mediated by its interaction with the ergosterol present in yeast and fungal membranes [69]. However, the molecular mechanisms at the base of this interaction are still object of a large debate. de Ghellinck et al. [59] investigated the activity of AmB on SLBs composed by natural lipids derived from Pichia Pastoris yeast [6], with a particular focus on the role of the native sterol components present in the SLBs. From the lipid analysis, ergosterol resulted to be the predominant sterol molecule in the Pichia Pastoris yeast extracts. NR data, collected in different contrast conditions and in presence or absence of sterols, were analysed according to the Abelès' matrix formalism [21] and the results of the analysis were expressed in terms of SLD profiles. Upon addition of AmB changes in these profiles were observed and their interpretation lead to the understanding of the action mechanism of AmB. In fact, in absence of sterols the changes indicated the insertion of $\mathrm{AmB}$ in the membrane as well as an accumulation of $\mathrm{AmB}$ molecules on the membrane surface (see Figures 10a-b and e-f). In presence of sterol (Figures 10c-d and g-h), the latter were removed from the membrane upon addition of AmB; the SLB final structure contained no sterol and a low amount of $\mathrm{AmB}$, while accumulation of $\mathrm{AmB}$ and sterols on the surface of the membrane was detected. These result represented the first structural investigation of $\mathrm{AmB}$ effects on yeast lipid membranes. The membranes reconstituted from natural lipid extracts reported in the work of de Ghellinck et al. [59] had a large degree of poly-unsaturation, that resulted crucial for the AmB interaction. In fact, the authors observed that $\mathrm{AmB}$ inserted into membranes even in the absence of sterols and that the inserted amount and the effect on the membrane structure critically depended on the lipid chain composition. The most important result they obtained was about the sterol depletion caused by AmB. In fact, AmB resulted to extract all the sterols from the SLBs, transferring them to a diffuse layer carpeting the surface of the membrane. This sterol depletion induced a significant membrane thinning that the authors related to the drug cytotoxicity. In fact, such a pronounced membrane thinning is likely to interfere with a broad range of membrane functions.

\section{Conclusions}

The collection of results reported in this chapter aimed to highlight the possibilities that an advanced scattering technique, as neutron reflectometry, can have for biologically relevant systems. As described in the previous sections, the data analysis approaches evolved to respond to the increase in samples complexity. Among the most relevant biological samples that can be investigated by NR, lipid membranes will continue to represent one of the most 

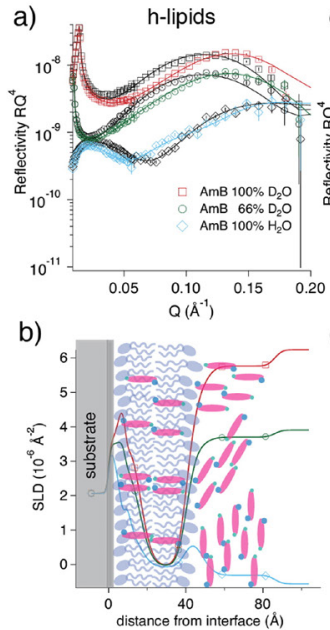
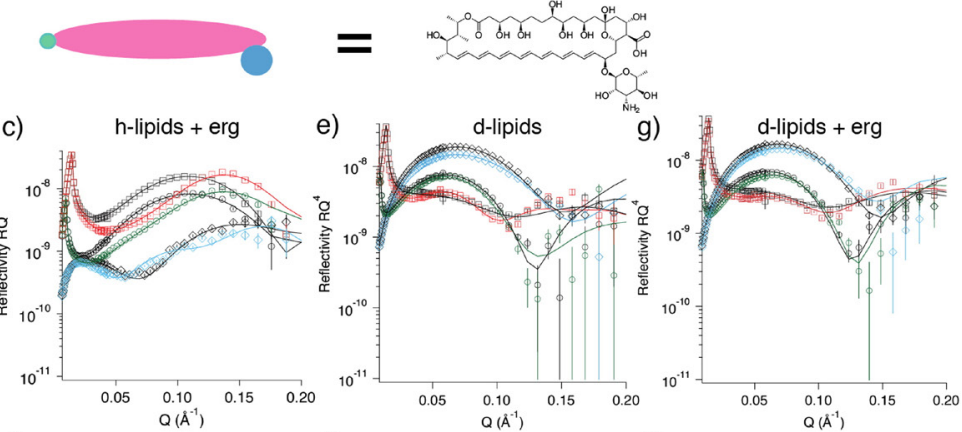

d)
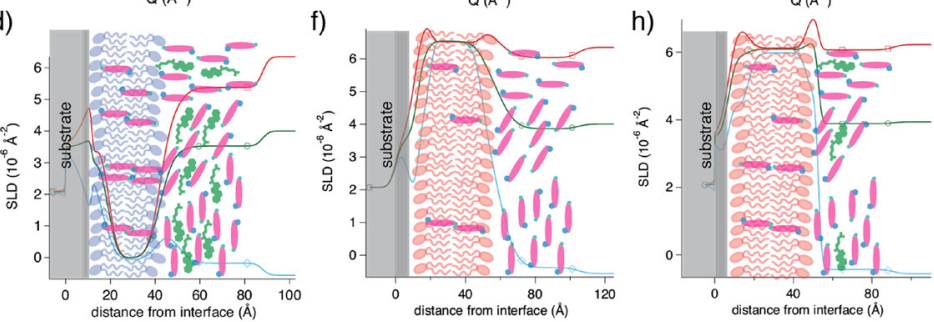

Figure 10. Reflectivity curves and corresponding SLD profiles illustrating the effect of AmB on SLBs composed P. pastoris lipid extracts in absence (a-b, e-f) and in presence (c-d, g-h) of the native sterols upon addition of $1 \mathrm{mM} \mathrm{AmB}$ in different contrast conditions (different $\mathrm{H}_{2} \mathrm{O}: \mathrm{D}_{2} \mathrm{O}$ ratio). The cartoons show the possible location of AmB corresponding to the layer thicknesses in the structures formed. Reprinted from Ref. [59], Copyright (2015), with permission from Elsevier.

relevant topics, because of their interest and implications in many aspects of our daily life. At the same time study of thin films in the context of novel bio-materials is expected to increase noticeably. In both cases, the use of natural biomolecules is expected to play a significant role. For this reason, new developments will be focused not only on the technique itself but mostly on sample production and preparation approaches. At the same time, the investigation of model systems will remain a hot topic in the physical chemistry and biophysics communities since NR allows to measure fundamental collective properties not accessible by means of other techniques.

\section{References}

[1] Z. Fisher, A. Jackson, A. Kovalevsky, E. Oksanen, H. Wacklin, in Exp. Methods Phys. Sci. (Academic Press, 2017), Vol. 49, pp. 1-75, ISBN 9780128053249

[2] M. Vaccaro, G. Mangiapia, L. Paduano, E. Gianolio, A. Accardo, D. Tesauro, G. Morelli, ChemPhysChem 8, 2526 (2007)

[3] R. Pignatello, T. Musumeci, L. Basile, C. Carbone, G. Puglisi, J. Pharm. Bioallied Sci. 3, 4 (2011)

[4] S. Mitragotri, D.G. Anderson, X. Chen, E.K. Chow, D. Ho, A.V. Kabanov, J.M. Karp, K. Kataoka, C.A. Mirkin, S.H. Petrosko et al., ACS Nano 9, 6644 (2015)

[5] T.L. Crowley, E.M. Lee, E.A. Simister, R.K. Thomas, Phys. B Phys. Condens. Matter 173, 143 (1991)

[6] A. de Ghellinck, H. Schaller, V. Laux, M. Haertlein, M. Sferrazza, E. Maréchal, H. Wacklin, J. Jouhet, G. Fragneto, PLoS One 9, e92999 (2014) 
[7] M. Moulin, G.A. Strohmeier, M. Hirz, K.C. Thompson, A.R. Rennie, R.A. Campbell, H. Pichler, S. Maric, V.T. Forsyth, M. Haertlein, Chem. Phys. Lipids 212, 80 (2018)

[8] S. Waldie, M. Moulin, L. Porcar, H. Pichler, G.A. Strohmeier, M. Skoda, V.T. Forsyth, M. Haertlein, S. Maric, M. Cárdenas, Sci. Rep. 9, 5118 (2019)

[9] M. Haertlein, M. Moulin, J.M. Devos, V. Laux, O. Dunne, V. Trevor Forsyth, in Methods Enzymol. (Academic Press, 2016), Vol. 566, pp. 113-157, ISBN 9780128030493

[10] M.C. Petty, W.A. Barlow, Film Deposition (Springer US, Boston, MA, 1990), pp. 93132, ISBN 978-1-4899-3716-2

[11] A.P. Girard-Egrot, L.J. Blum, in Nanobiotechnology Biomim. Membr. (Springer US, Boston, MA, 2007), pp. 23-74

[12] H.P. Wacklin, Langmuir 27, 7698 (2011)

[13] H. Mohwald, Annu. Rev. Phys. Chem. 41, 441 (1990)

[14] C. Schladitz, E.P. Vieira, H. Hermel, H. Möhwald, Biophys. J. 77, 3305 (1999)

[15] U. Mennicke, T. Salditt, Langmuir 18, 8172 (2002)

[16] S. Micciulla, D.W. Hayward, Y. Gerelli, A. Panzarella, R. von Klitzing, M. Gradzielski, L. Chiappisi, Commun. Chem. 2, 61 (2019)

[17] Y. Gerelli, L. Porcar, G. Fragneto, Langmuir 28, 15922 (2012)

[18] V. Rondelli, P. Brocca, N. Tranquilli, G. Fragneto, E. Del Favero, L. Cantù, Biophys. Chem. 229, 135 (2017)

[19] G. Fragneto, T. Charitat, J. Daillant, Eur. Biophys. J. 41, 863 (2012)

[20] V. Rondelli, G. Fragneto, S. Motta, E.D. Favero, L. Cantù, J. Phys. Conf. Ser. 340, 012083 (2012)

[21] F. Abelès, Ann. Phys. (Paris). 12, 706 (1950)

[22] L.G. Parratt, Phys. Rev. 95, 359 (1954)

[23] R.A. Campbell, Y. Saaka, Y. Shao, Y. Gerelli, R. Cubitt, E. Nazaruk, D. Matyszewska, M.J. Lawrence, J. Colloid Interface Sci. 531, 98 (2018)

[24] Y. Gerelli, Phys. Rev. Lett. 122, 248101 (2019)

[25] Y. Gerelli, J. Appl. Crystallogr. 49, 330 (2016)

[26] A. Nelson, J. Appl. Crystallogr. 39, 273 (2006)

[27] M. Björck, G. Andersson, J. Appl. Crystallogr. 40, 1174 (2007), 1503.07163

[28] A.V. Hughes, F. Ciesielski, A.C. Kalli, L.A. Clifton, T.R. Charlton, M.S.P. Sansom, J.R.P. Webster, Acta Crystallogr. Sect. D Struct. Biol. 72, 1227 (2016)

[29] A.R. Nelson, S.W. Prescott, J. Appl. Crystallogr. 52, 193 (2019)

[30] E. Schneck, I. Berts, A. Halperin, J. Daillant, G. Fragneto, Biomaterials 46, 95 (2015)

[31] V.M. Latza, I. Rodriguez-Loureiro, I. Kiesel, A. Halperin, G. Fragneto, E. Schneck, Langmuir 33, 12708 (2017)

[32] S. Micciulla, Y. Gerelli, R.A. Campbell, E. Schneck, Langmuir 34, 789 (2018)

[33] S. Micciulla, Y. Gerelli, E. Schneck, Biophys. J. 116, 1259 (2019)

[34] M. Wiener, S. White, Biophys. J. 61, 434 (1992)

[35] N. Kučerka, J.F. Nagle, J.N. Sachs, S.E. Feller, J. Pencer, A. Jackson, J. Katsaras, Biophys. J. 95, 2356 (2008)

[36] M. Belička, Y. Gerelli, N. Kučerka, G. Fragneto, Soft Matter 11, 6275 (2015)

[37] J. Pan, X. Cheng, F.A. Heberle, B. Mostofian, N. Kučerka, P. Drazba, J. Katsaras, J. Phys. Chem. B 116, 14829 (2012)

[38] N. Kučerka, B.W. Holland, C.G. Gray, B. Tomberli, J. Katsaras, J. Phys. Chem. B 116, $232(2012)$

[39] L. Tavagnacco, Y. Gerelli, A. Cesàro, J.W. Brady, J. Phys. Chem. B 120, 9987 (2016) 
[40] P.c. Chen, R. Shevchuk, F.M. Strnad, C. Lorenz, L. Karge, R. Gilles, A.M. Stadler, J. Hennig, J.S. Hub, J. Chem. Theory Comput. 15, 4687 (2019)

[41] A.A. Mungikar, D. Forciniti, Computer simulations and neutron reflectivity of proteins at interfaces (2002)

[42] C.F. Majkrzak, N.F. Berk, S. Krueger, J.A. Dura, M. Tarek, D. Tobias, V. Silin, C.W. Meuse, J. Woodward, A.L. Plant, Biophys. J. 79, 3330 (2000)

[43] S. Bobone, Y. Gerelli, M. De Zotti, G. Bocchinfuso, A. Farrotti, B. Orioni, F. Sebastiani, E. Latter, J. Penfold, R. Senesi et al., Biochim. Biophys. Acta - Biomembr. 1828, 1013 (2013)

[44] A. Koutsioubas, J. Phys. Chem. B 120, 11474 (2016)

[45] F. Heinrich, M. Lösche, Biochim. Biophys. Acta - Biomembr. 1838, 2341 (2014)

[46] A.R. McCluskey, J. Grant, A.J. Smith, J.L. Rawle, D.J. Barlow, M.J. Lawrence, S.C. Parker, K.J. Edler, J. Phys. Commun. 3, 075001 (2019)

[47] L. Darré, J. Iglesias-Fernandez, A. Kohlmeyer, H. Wacklin, C. Domene, J. Chem. Theory Comput. 11, 4875 (2015)

[48] W. Humphrey, A. Dalke, K. Schulten, J. Mol. Graph. 14, 33 (1996)

[49] K. Simons, E. Ikonen, Nature 387, 569 (1997)

[50] K. Simons, W.L. Vaz, Annu. Rev. Biophys. Biomol. Struct. 33, 269 (2004)

[51] K. Simons, M.J. Gerl, Nat. Rev. Mol. Cell Biol. 11, 688 (2010)

[52] P.L. Yeagle, FASEB J. 3, 1833 (1989)

[53] G. Fragneto, Eur. Phys. J. Spec. Top. 213, 327 (2012)

[54] T. Salditt, C. Münster, U. Mennicke, C. Ollinger, G. Fragneto, Langmuir 19, 7703 (2003)

[55] J. Daillant, E. Bellet-Amalric, A. Braslau, T. Charitat, G. Fragneto, F. Graner, S. Mora, F. Rieutord, B. Stidder, Proc. Natl. Acad. Sci. U. S. A. 102, 11639 (2005)

[56] Y. Gerelli, L. Porcar, L. Lombardi, G. Fragneto, Langmuir 29, 12762 (2013)

[57] V. Rondelli, E. Del Favero, S. Motta, L. Cantù, G. Fragneto, P. Brocca, Eur. Phys. J. E 36, 73 (2013)

[58] G. Fragneto, R. Delhom, L. Joly, E. Scoppola, Curr. Opin. Colloid Interface Sci. 38, 108 (2018)

[59] A. de Ghellinck, G. Fragneto, V. Laux, M. Haertlein, J. Jouhet, M. Sferrazza, H. Wacklin, Biochim. Biophys. Acta - Biomembr. 1848, 2317 (2015)

[60] L.A. Clifton, S.A. Holt, A.V. Hughes, E.L. Daulton, W. Arunmanee, F. Heinrich, S. Khalid, D. Jefferies, T.R. Charlton, J.R. Webster et al., Angew. Chemie - Int. Ed. 54, 11952 (2015)

[61] J.M. Pagès, C.E. James, M. Winterhalter, The porin and the permeating antibiotic: A selective diffusion barrier in Gram-negative bacteria (2008)

[62] A.P. Le Brun, L.A. Clifton, C.E. Halbert, B. Lin, M. Meron, P.J. Holden, J.H. Lakey, S.A. Holt, Biomacromolecules 14, 2014 (2013)

[63] M. Vaara, Microbiol. Rev. 56, 395 (1992)

[64] A.R. Mazzer, L.A. Clifton, T. Perevozchikova, P.D. Butler, C.J. Roberts, D.G. Bracewell, J. Chromatogr. A 1499, 118 (2017)

[65] J.M. Harris, ed., Poly(Ethylene Glycol) Chemistry (Springer US, Boston, MA, 1992), ISBN 978-1-4899-0705-9

[66] H. Chen, L. Yuan, W. Song, Z. Wu, D. Li, Prog. Polym. Sci. 33, 1059 (2008)

[67] R.S. Harrison, P.C. Sharpe, Y. Singh, D.P. Fairlie, in Rev. Physiol. Biochem. Pharmacol. (Springer Berlin Heidelberg, Berlin, Heidelberg, 2007), Vol. 159, pp. 1-77 
[68] A. Martel, L. Antony, Y. Gerelli, L. Porcar, A. Fluitt, K. Hoffmann, I. Kiesel, M. Vivaudou, G. Fragneto, J.J. de Pablo, J. Am. Chem. Soc. 139, 137 (2017)

[69] K.C. Gray, D.S. Palacios, I. Dailey, M.M. Endo, B.E. Uno, B.C. Wilcock, M.D. Burke, Proc. Natl. Acad. Sci. 109, 2234 (2012) 